\title{
KIDNEY'S FUNCTIONAL STATE ASSESSMENT WHEN USING DIFFERENT REGIMENS OF INTRAOPERATIVE FLUID THERAPY
}

\author{
Yanina Morozova, Aleksandr Pavlov
}

The aim of the research. To study the kidneys functional state when using different regimens of intraoperative fluid therapy in high cardiac risk patients during abdominal surgery.

Materials and methods. 142 patients who underwent abdominal surgical interventions mainly for oncoproctological diseases of the gastrointestinal tract, aged over 50 years old and with a history of stable coronary heart disease were divided into four groups depending on the way of intraoperative fluid therapy, which was performed according to two regimens: restrictive $(R)$ and liberal $(L) . R 1(n=36)$ with rate of intraoperative fluid therapy $3-5 \mathrm{ml} / \mathrm{kg} / \mathrm{h}, R 2(\mathrm{n}=35)$ patients received $5-8 \mathrm{ml} / \mathrm{kg} / \mathrm{h}$ during surgery, $L 1(n=35)$ with intraoperative fluid rate of $8-11 \mathrm{ml} / \mathrm{kg} / \mathrm{h}$ and $\mathrm{L} 2(\mathrm{n=} 36)$ - more than $11 \mathrm{ml} / \mathrm{kg} / \mathrm{h}$ intraoperatively. The study of the functional state of the kidneys included the determination of such indicators as urea, creatinine, diuresis, the degree of AKI according to KDIGO in two stages of the study - before surgery and 18-24 hours after.

Results. The greatest tendency to develop acute kidney injury was observed in R1 subgroup with a restrictive intraoperative fluid therapy regimen, and the smallest in L1 subgroup with a relatively liberal regimen. The R2 and L2 subgroups took an intermediate place in the number of renal complications. A high tendency to develop renal dysfunction in patients of RI subgroup was associated with circulatory hypokinesia and a moderate decrease of renal perfusion.

Conclusions. The study found that restriction of infusion in R1 subgroup contributed to the development of renal dysfunction in almost half of the patients. First of all it was associated with a decrease of GFR in conditions of circulatory hypokinesia, which is larger in R1 subgroup and amounted to about $35 \%$. The safest regimens of intraoperative fluid therapy in relation to renal function in the perioperative period were relatively liberal (subgroup L1) and relatively restrictive (subgroup $R 2$ ), which provided the least number of complications in patients Keywords: fluid therapy, restrictive, liberal, acute kidney injury, glomerular filtration rate

How to cite:

Morozova, Y., Pavlov, A. (2021). Kidney`s functional state assessment when using different regimens of intraoperative fluid therapy. ScienceRise: Medical Science, 6 (45), 30-33. doi: http://doi.org/10.15587/2519-4798.2021.247973

(C) The Author(s) 2021

This is an open access article under the Creative Commons CC BY license hydrate

\section{Introduction}

Kidney`s functional state assessment under the influence of many factors of the perioperative period is a current trend in modern anesthesiology [1]. Surgical trauma, blood loss, hemodynamic instability and sympathoadrenal activity lead to a decrease of glomerular filtration and to the significant release of biologically active substances that have a damage effect on kidney cells (cytokines, peroxide radicals, iron ions, etc.). Acute kidney injury is one of the complications of the perioperative period, which is characterized by a sudden loss or insufficiency of renal function, which leads to the accumulation of urea and other nitrogenous compounds in the body $[2,3]$. The incidence of acute kidney damage varies from 1.9 to $18 \%$ depending on the performed surgery [4, 5]. Intraoperative fluid balance is one of the modifiable risk factors for acute kidney injury [6]. At the same time an adequate fluid therapy is absolutely essential to prevent prerenal acute kidney damage [7].

The aim of the research. To study the kidneys functional state when using different regimens of in- traoperative fluid therapy in high cardiac risk patients during abdominal surgery.

\section{Materials and methods}

The single-center, prospective, randomized study included 142 patients who were treated at the "Kharkov City Clinical Hospital No. 2", and "V.T. Zaitsev Institute of General and Urgent Surgery of National Academy of Medical Sciences of Ukraine" for the period 2017-2021, who underwent abdominal surgical interventions mainly for oncoproctological diseases of the gastrointestinal tract, aged from 50 to 75 years (the mean age $66.1 \pm 0.7$ years) old and with a history of stable coronary heart disease.

The work was carried out in accordance with the Code of Ethics of the World Medical Association (Helsinki Declaration). All patients included in the study provided written informed consent. The work was approved by the Commission on Bioethics of the Kharkiv Medical Academy of Postgraduate Education, protocol No. 3 $10 / 12 / 2021$. 
By the method of blind randomization, patients were divided into two groups depending on the way of intraoperative fluid therapy, which was performed according to two regimens: restrictive and liberal. Patients were divided into such groups: the first group with restrictive (R) regimen of intraoperative fluid therapy $(n=71)$ included patients with rate of intraoperative fluid therapy not more than $8 \mathrm{ml} / \mathrm{kg} / \mathrm{h}$. Patients in this group were divided into two subgroups: $\mathrm{R} 1(\mathrm{n}=36)$ with rate of intraoperative fluid therapy $3-5 \mathrm{ml} / \mathrm{kg} / \mathrm{h}, \mathrm{R} 2(\mathrm{n}=35)$ patients received $5-8 \mathrm{ml} / \mathrm{kg} / \mathrm{h}$ during surgery. The second group with a liberal (L) regimen of intraoperative fluid therapy $(n=71)$ included patients with rate of intraoperative fluid therapy more than $8 \mathrm{ml} / \mathrm{kg} / \mathrm{h}$. Patients in this group were divided into two subgroups: L1 $(\mathrm{n}=35)$ with intraoperative fluid rate of $8-11 \mathrm{ml} / \mathrm{kg} / \mathrm{h}$ and L2 $(\mathrm{n}=36)-$ more than $11 \mathrm{ml} / \mathrm{kg} / \mathrm{h}$ intraoperatively. The study of the functional state of the kidneys included the determination of such indicators as urea, creatinine, diuresis in two stages of the study - before surgery and 18-24 hours after. Determination of urea and creatinine levels was performed on a biochemical automatic analyzer DS-26, the GRF was calculated by Cockcroft-Gault formula. Cases of kidney's dysfunction were determined by KDIGO Clinical Practice Guideline for Acute Kidney Injury [8].

Statistical processing of data was performed via STATISTICA 6,0 program by parametric and nonparametric methods of Wilcoxon (for comparison in the dynamics of patients in one group) and Mann-Whitney (to assess differences between groups). For all types of analysis, the differences were statistically significant at $\mathrm{p}<0.05$.

\section{Results}

Many of the patients enrolled in this study had preoperative risk factors for acute kidney injury. Among them should be noted some of them: the age of patients, the presence of concomitant cardiac pathology (coronary heart disease, heart failure, hypertension), oncology disease. As shown in Table 1, a decrease in the functional kidney`s reserves in the examined patients compared with the initial state was manifested in a decrease of GFR to $55-70 \mathrm{ml} / \mathrm{min}$, at the same time serum creatinine and urea values were within normal limits in most patients.

Table 1

Parameters of kidney`s functional state $(\mathrm{M} \pm \mathrm{m})$

\begin{tabular}{|c|c|c|c|c|c|c|c|c|}
\hline \multirow{2}{*}{ Parameter } & \multicolumn{4}{|c|}{ Before surgery } & \multicolumn{4}{|c|}{ After surgery(in 18-24 hours) } \\
\hline & $\mathrm{R} 1$ & $\mathrm{R} 2$ & L1 & $\mathrm{L} 2$ & $\mathrm{R} 1$ & $\mathrm{R} 2$ & L1 & L2 \\
\hline Urea, mmol/l & $5.2 \pm 0.2$ & $5.4 \pm 0.2$ & $5.1 \pm 0.2$ & $5.3 \pm 0.2$ & $5.7 \pm 0.2$ & $5.8 \pm 0.2$ & $5.7 \pm 0.2$ & $5.8 \pm 0.1$ \\
\hline $\begin{array}{l}\text { Creatinine, } \mathrm{mg} / \mathrm{dl} \\
\mathrm{n}(0.04-0.11)\end{array}$ & $\begin{array}{c}0.07 \pm \\
\pm 0.003\end{array}$ & $\begin{array}{c}0.07 \pm \\
\pm 0.002\end{array}$ & $\begin{array}{c}0.08 \pm \\
\pm 0.002\end{array}$ & $\begin{array}{c}0.07 \pm \\
\pm 0.002\end{array}$ & $\begin{array}{c}0.13 \pm \\
\pm 0.003^{1}\end{array}$ & $\begin{array}{c}0.09 \pm \\
\pm 0.001\end{array}$ & $\begin{array}{c}0.09 \pm 0 \\
\pm .001\end{array}$ & $\begin{array}{c}0.09 \pm \\
\pm 0.001\end{array}$ \\
\hline Diuresis, ml. & $\mathrm{N}$ & $\mathrm{N}$ & $\mathrm{N}$ & $\mathrm{N}$ & $2346.2 \pm 143.9$ & $2672 \pm 183.9$ & $2575 \pm 175$ & $2842.3 \pm 168.3$ \\
\hline Diuresis rate, $\mathrm{ml} / \mathrm{kg} / \mathrm{h}$ & $\mathrm{N}$ & $\mathrm{N}$ & $\mathrm{N}$ & $\mathrm{N}$ & $0.9 \pm 0.3$ & $1.2 \pm 0.2$ & $1.2 \pm 0.3$ & $1.3 \pm 0.3$ \\
\hline $\mathrm{GFR}, \mathrm{ml} / \mathrm{min} / 1,73 \mathrm{~m}^{2}$ & $85.5 \pm 8.2$ & $87.0 \pm 6.5$ & $80.3 \pm 6.2$ & $82.1 \pm 4.2$ & $55.9 \pm 3.5^{1}$ & $69.2 \pm 3.7$ & $64.2 \pm 4.0$ & $59.7 \pm 2.9$ \\
\hline
\end{tabular}

Note: ${ }^{\prime}$ significant difference between indicators of Rl and other groups ( $\left.p<0.05\right), M$-average; $m$ - the standard error of the mean

In patients of the 1 st subgroup with a restrictive regimen of intraoperative fluid therapy a significant GFR`s decrease and an increase blood creatinine level was observed in the early postoperative period. This indicates significant changes in the functional kidney`s status in these patients. A more detailed analysis of the KDIGO Acute Renal Damage Scale revealed changes corresponding to stage $1 \mathrm{AKI}$ in 5 patients (14\%), stage 2 in 9 patients $(25 \%)$ and stage 3 in 1 patient ( $3 \%$ of the total number in the subgroup). These changes occurred despite adequate correction of hemodynamic parameters with maintenance of the average blood pressure in the range of $80-90 \mathrm{~mm} \mathrm{Hg}$. This level is optimal to ensure adequate renal perfusion and reduce renal complications in patients with hypertension [9]. A possible explanation for this fact is that patients in subgroup 1 developed a tendency to circulatory hypokinesia, which more often than others required the introduction of sympathomimetic drugs to support hemodynamics.

Patients of the 2nd subgroup with a relatively restrictive regimen of intraoperative fluid therapy showed similar changes in renal parameters, but they were less evident compared to the 1 st subgroup. The decrease in the average GFR was about $26 \%$, while in the 1 st sub- group $-35 \%$. The creatinine blood level significantly increased, but within normal values. Cases of $1^{\text {st }}$ stage acute kidney injury noted in 6 patients (17\% of the number in the subgroup).

In patients with a liberal regimen of intraoperative fluid therapy was a similar dynamics of kidney function in the perioperative period. In patients of subgroup L1 with a relatively liberal fluid regimen, a moderate increase in creatinine and urea blood levels were within the reference values. The GFR's reduction was about $20 \%$. Signs of the first stage of acute kidney injury according to KDIGO classification in subgroup L1 were found in 3 patients $(9 \%)$. In patients with a liberal regimen of intraoperative fluid therapy (subgroup L 2) were found the same changes in renal parameters with a decrease of GFR by $20 \%$. Signs of renal dysfunction of the first stage were found in 6 patients $(17 \%)$, 2nd stage - in the 1 patient (6\% of the number in the subgroup).

The Fig. 1 shows that the greatest predisposition to the development of acute kidney injury was observed in subgroup R1 with a restrictive regimen of intraoperative fluid therapy, the lowest - in subgroup L1 with a relatively liberal. Subgroups R2 and L2 took an intermediate position in the number of renal complications. 




Fig. 1. Cases of acute kidney injury in subgroups

The highest predisposition to the development of renal dysfunction in patients of subgroup $\mathrm{R} 1$ was associated with hypokinesia of blood circulation and a moderate decrease in renal perfusion. For all those patients who had a decrease in hourly diuresis and GFR in the second half of the operation and in the early postoperative period the fluid therapy were optimized within a daily fluid intake of 20-25 $\mathrm{ml} / \mathrm{kg}$. If these measures were ineffective, diuretics were additionally administered (torasemide $20-40 \mathrm{mg}$ until sufficient hourly diuresis was achieved). All these measures led to increase in the fluid load of patients while maintaining a "zero" or weakly positive fluid balance per day. Similar measures to correct the kidney`s functional status, if necessary, were used in other groups of patients.

\section{Discussion}

Thus, the renal dysfunctions of varying severity naturally occur in many high cardiac risk patients during surgery [10]. This is facilitated by both surgical trauma (the impact of stress reactions) and the presence of many risk factors in patients [11]. Differences found during the perioperative period due to the development of renal dysfunction in different subgroups of patients suggest that intraoperative fluid therapy also significantly affects the functional state of the kidneys. Restriction of fluid supply in the R1 subgroup contributed to the development of renal dysfunction in almost half of the patients. This is primarily due to a decrease in GFR in conditions of circulatory hypokinesia, which in the subgroup R1 was the largest and amounted to about $35 \%$. These patients in the early postoperative period need to increase the rate of fluids and the use of diuretics with a significant reduction in hourly diuresis. The implementation of these measures was sufficient to restore adequate diuresis and stabilize renal function. None of the patients in the subsequent postoperative period showed signs of progression of renal failure.

Study limitations. Restrictions due to the involvement of small sample of patients.

Prospects for future research. It would be interesting to examine younger patients to find out their reaction on fluid load. Also, interesting to investigate patients with other functional classes of chronic cardiac failure.

\section{Conclusion}

1. The highest predisposition to the development of renal dysfunction in patients of subgroup R1 was associated, in our opinion, with hypokinesia of blood circulation and a moderate decrease in renal perfusion.

2 . The study found that both restriction of fluid and fluid overload during abdominal surgeries for high cardiac risk patients are potentially dangerous in terms of the development of renal dysfunction.

3. The safest regimens for intraoperative fluid therapy with respect to renal function in the perioperative period were relatively liberal (subgroup L1) and relatively restrictive (subgroup R2), which provided the least number of complications in patients.

\section{Conflict of interests}

The authors declare that they have no conflicts of interest.

\section{Financing}

The study was performed without financial support.

\section{Acknowledgments}

We express our gratitude to the patients who took part in the study, as well as the specialists who contributed to the investigation.

\section{References}

1. Kim, B. R., Yoon, S., Song, G. Y., Lee, S., Bahk, J. H., Nam, K. (2021). The impact of total intravenous anesthesia versus inhalation anesthesia on acute kidney injury after major abdominal surgery: a propensity score analysis. Journal of Anesthesia, 35 (1), 112-121. doi: https://doi.org/10.1007/s00540-020-02882-9 
2. Nishimoto, M., Murashima, M., Kokubu, M., Matsui, M., Eriguchi, M., Samejima. et. al. (2021). External Validation of a Prediction Model for Acute Kidney Injury Following Noncardiac Surgery. JAMA Network Open, 4 (10). doi: https://doi.org/10.1001/ jamanetworkopen.2021.27362

3. Miller, T. E., Mythen, M., Shaw, A. D., Hwang, S., Shenoy, A. V., Bershad, M. et al. (2021). Association between perioperative fluid management and patient outcomes: a multicentre retrospective study. British Journal of Anaesthesia, 126 (3), $720-729$. doi: https://doi.org/10.1016/j.bja.2020.10.031

4. Zhao, B. C., Lei, S.H., Yang, X., Zhang, Y., Qiu, S. D., Liu, W. F. et. al. (2021). Assessment of prognostic value of intraoperative oliguria for postoperative acute kidney injury: a retrospective cohort study. British Journal of Anaesthesia, 126 (4), 799 807. doi: https://doi.org/10.1016/j.bja.2020.11.018

5. Guangyu, Y., Jingfeng, L., Xing, L., Hong, Y., Yao, L. (2021). Cardio- and Cerebrovascular Outcomes of Postoperative Acute Kidney Injury in Noncardiac Surgical Patients With Hypertension. Frontiers in Pharmacology, 12. doi: https://doi.org/10.3389/ fphar.2021.696456

6. Yunos, N. M., Bellomo, R., Glassford, N., Sutcliffe, H., Lam, Q., Bailey, M. (2014). Chloride-liberal vs. chloriderestrictive intravenous fluid administration and acute kidney injury: an extended analysis. Intensive Care Medicine, 41 (2), $257-264$. doi: https://doi.org/10.1007/s00134-014-3593-0

7. Essber, H., Cohen, B., Artis, A. S., Leung, S. M., Maheshwari, K., Khan, M. Z. et. al. (2021). Renal injury after open versus laparoscopic non-cardiac surgery: a retrospective cohort analysis. Brazilian Journal of Anesthesiology, 71 (1), 50-57. doi: https://doi.org/10.1016/j.bjane.2020.12.008

8. Kidney Disease Improving Global Outcomes (KDIGO) Acute Kidney Injury Work Group (2012). KDIGO clinical practice guideline for acute kidney injury. Kidney Int Suppl, 2 (1), 138.

9. Prowle, J. R., Forni, L. G., Bell, M., Chew, M. S., Edwards, M., Grams, M. E. et. al. (2021). Postoperative acute kidney injury in adult non-cardiac surgery: joint consensus report of the Acute Disease Quality Initiative and PeriOperative Quality Initiative. Nature Reviews Nephrology, 17 (9), 605-618. doi: https://doi.org/10.1038/s41581-021-00418-2

10. Park, S., Cho, H., Park, S., Lee, S., Kim, K., Yoon, H. J. et. al. (2018). Simple Postoperative AKI Risk (SPARK) Classification before Noncardiac Surgery: A Prediction Index Development Study with External Validation. Journal of the American Society of Nephrology, 30 (1), 170-181. doi: https://doi.org/10.1681/asn.2018070757

11. Meersch, M., Schmidt, C., Zarbock, A. (2017). Perioperative Acute Kidney Injury. Anesthesia Analgesia, 125 (4), 1223 1232. doi: https://doi.org/10.1213/ane.0000000000002369

Received date 07.09.2021

Accepted date 14.10.2021

Published date 30.11.2021

Yanina Morozova, Assistant, Department of Anesthesiology, Intensive Care, Transfusiology and Hematology, Kharkiv Medical Academy of Postgraduate Education, Amosova str., 58, Kharkiv, Ukraine, 61176

Aleksandr Pavlov, Doctor of Medical Sciences, Professor, Head of Department, Department of Anesthesiology, Intensive Care, Transfusiology and Hematology, Kharkiv Medical Academy of Postgraduate Education, Amosova str., 58, Kharkiv, Ukraine, 61176

*Corresponding author: Yanina Morozova, e-mail:dr.yaninamorozova@gmail.com 